\title{
Temporal organization of pattern structure
}

\author{
STEPHEN K. REED and JAMES L. BROWN \\ Case Western Reserve University, Cleveland, Ohio 44106
}

\begin{abstract}
Two pattern reproduction experiments examined the relations among the figural goodness of a pattern, the organization of two parts within the pattern, and the interpart interval (ISI), which ranged from 40 to $200 \mathrm{msec}$. If the parts contained connected line segments, performance was slightly better (3\%-5\% gain in accuracy) at a $40-\mathrm{msec}$ ISI than at a $200-\mathrm{msec}$ ISI. If the parts contained unconnected line segments, reproduction accuracy of the first part declined sharply between 40 and 200 msec. These results were interpreted by assuming that the parts were perceived as a single whole pattern at a $40-\mathrm{msec}$ ISI but as two separate patterns at a 200-msec ISI. One surprising finding, the lack of an interaction between figural goodness and ISI, was explained in terms of a response bias in favor of figurally good patterns. A secondary manipulation revealed that a part was more accurately reproduced in a good figure context than in a poor figure context but was most accurately reproduced when it appeared alone.
\end{abstract}

The structural organization of patterns, emphasized by Gestalt psychology, continues to attract the attention of psychologists. Experiments on pattern structure are summarized by Garner (1974), and the relation of structural theories to other theories of pattern recognition is discussed by Reed (1978). The present series of experiments was motivated by Palmer's (1977) research on how the organization of a pattern into component parts affects the synthesis of the parts. Subjects in Palmer's experiment saw two parts, each consisting of three straight lines connecting dots in a 3 by 3 dot matrix. The two dot matrices were adjacent and the task was to synthesize the two parts into a whole pattern. Palmer found that about $1.5 \mathrm{sec}$ were needed to synthesize the parts if they had a high level of organization, but about $4.0 \mathrm{sec}$ were needed if the parts had a medium or low level of organization. The high level of organization was the most natural way of organizing the patterns according to both subjects' ratings and Palmer's theoretical predictions.

One way of extending the paradigm used by Palmer (1977) would be to ask subjects to draw a pattern from two parts that occurred sequentially rather than simultaneously. Both Haber (1978) and Hochberg (1978) have recently emphasized the importance of integrating successive glances in forming a description of a pattern. Haber argues that since the resolution of fine detail is only possible in the center of the visual field, several glances are necessary to achieve a clear representation of the visual scene. Also, since much of perception occurs while the head and body are in motion, the continuous transformations of the visual field have to be integrated into a coherent percept.

Requests for reprints should be sent to Stephen K. Reed, Department of Psychology, Case Western Reserve University, Cleveland, Ohio 44106. We appreciate the comments of Grover C. Gilmore on an earlier draft of the manuscript.
Hochberg has argued that the problem of how to combine successive sensory input is central to the study of vision, hearing, and touch. In the case of visual perception, Hochberg shows how the integration of successive glimpses is necessary both for the active viewing of stationary objects and the perception of motion pictures.

The current experiments investigate the temporal organization of pattern structure by using a modification of the sequential part technique designed by McFarland (1965). McFarland successively presented either the three sides or the three angles of a triangle. Each side was presented for $10 \mathrm{msec}$, and the subject's task was to judge whether the parts appeared as simultaneous, overlapping, or successive. The percent "simultaneous" judgments declined to $0 \%$ as the interpart interval (ISI) increased to $100 \mathrm{msec}$, and the percent "successive" judgments increased to almost $100 \%$ as the ISI increased to $200 \mathrm{msec}$. Similar results were found for presenting the three angles, except that the parts appeared joined more often when the sides were presented rather than angles.

The stimuli used in the present experiments were similar to the line patterns used by Palmer (1977). The two parts were sequentially presented in a tachistoscope, separated by dark intervals ranging from 40 to $200 \mathrm{msec}$. The task required that the subjects draw the entire sixsegment pattern and mark the three segments that occurred in the first exposure. Our expectations were that subjects would perceive the pattern less as a whole and more as two separate parts as the ISI increased.

The primary purpose of our study was to investigate whether various structural characteristics of patterns would interact with the temporal separation of the parts. We distinguish between two kinds of structural characteristics. The first refers to the figural goodness of the whole pattern and is the major independent variable in the experiments reviewed by Garner (1974). 
The second refers to the figural goodness or organization of the two parts within the whole and is the major independent variable investigated by Palmer (1977). Figure 4 shows this distinction. The pattern in the first row was rated high in goodness and the pattern in the second row was rated low in goodness. The patterns in the left column have a high level of organization since they are divided into natural parts. The patterns in the right column have a low level of organization since they are divided into unnatural parts. The experiments were designed to test the hypothesis that the figural goodness of the whole pattern would influence performance more at short ISIs, and the organization of the parts would influence performance more at long ISIs.

\section{EXPERIMENT 1}

Previous research has demonstrated that it is easier to encode and reproduce patterns scoring high on figural goodness than patterns scoring low on figural goodness (see Garner, 1974). Experiment 1 examined the effect of figural goodness on a reproduction task as the ISI was varied. If the pattern is perceived as a whole, the figural goodness of the whole should influence performance. If the pattern is perceived as two separate parts, performance should be influenced more by the figural goodness and organization of the parts within the pattern. A second issue explored in Experiment 1 was the effect of context on perception. If high- and low-goodness pairs share a common part, it is possible to study whether the same part is reproduced more accurately when it occurs in a high-goodness $(\mathrm{H})$ pattern than when it occurs in a low-goodness (L) pattern.

\footnotetext{
Method

Stimuli. Two scaling sessions were conducted to scale the figural properties of the stimuli used in Experiment 1 . Subjects in both studies were undergraduates at Case Western Reserve University who were tested in a single group session. The first study asked 32 subjects to judge the figural goodness of 54 six-segment patterns, constructed by connecting dots in a 3 by 3 matrix. The patterns consisted of 27 pairs that shared a common three-segment part. The patterns were drawn in an attempt to make one member of a pair a figurally good pattern and the other member a figurally bad pattern. Following the procedure used by Garner and Clement (1963), subjects were instructed to rate each pattern on a 7-point scale ranging from 1 for the best (well structured) patterns to 7 for the poorest (poorly structured) patterns. The patterns were photocopied to form test booklets, and subjects were allowed to work at their own pace. After completing the first part of the task, subjects divided each pattern into two mutually exclusive three-segment parts, chosen to be the "best" or the "most natural" parts within the pattern. Their divisions were used to select patterns for Experiment 2.

The average figural goodness ratings were used to select the 12 pairs that best satisfied the criterion that one member of a pair have a low rating and one member have a high rating. Figure 1 shows the 12 pairs, divided into two three-segment parts to illustrate that each pair of patterns shared a common part. The 24 patterns were used in Experiment 1.
}
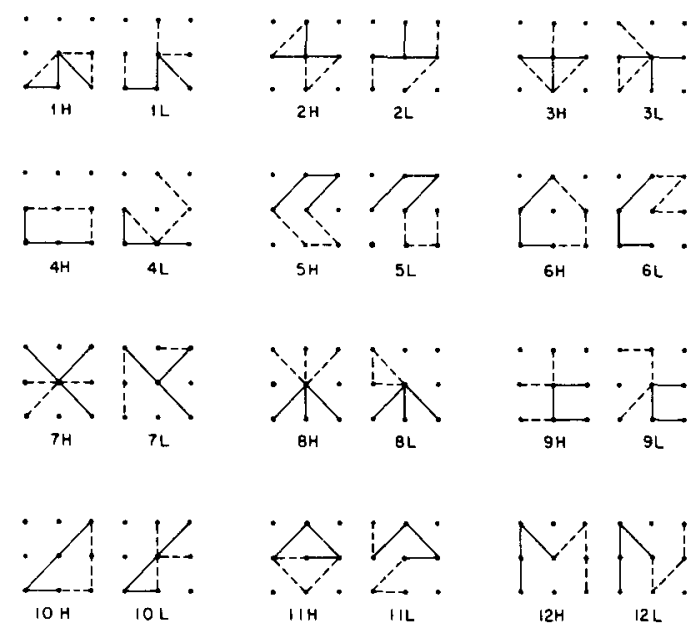

Figure 1. Patterns used in Experiment 1.

The purpose of the second scaling session was to scale the figural goodness of each part and measure the organization of the parts within the whole. Ten undergraduates received test booklets containing the 36 parts shown in Figure 1. They were instructed to rate each of the three-segment parts on a 7-point scale of figural goodness. The 36 parts were presented in a random order and were not shown in the context of the whole pattern. The second half of the task used the technique of Palmer $(1977)$ to measure the organization of the parts within the whole. Each of the 24 patterns was divided into the two parts shown in Figure 1. Each item consisted of three dot matrices with the whole pattern in the left matrix and the two parts in the other two matrices. Subjects were asked to judge how obvious the parts were within the pattern. Ratings were made on an 11-point scale ranging from 0 for a very bad organization to 10 for a very good organization. Table 1 shows the average ratings obtained in the two scaling sessions.

The average figural goodness ratings of $H$ patterns was 2.0, and the average figural goodness ratings of the $L$ patterns was 5.1. The $H$ and $L$ patterns did not differ significantly in the figural goodness ratings assigned to their parts or in the organization measure of how well the two parts fit within the whole pattern. The average rating of the parts that differed in each pair was 3.3 for $\mathrm{H}$ patterns and 3.7 for $\mathrm{L}$ patterns $[\mathrm{t}(22)=.83]$. The average organization rating was 6.3 for $\mathrm{H}$ patterns and 5.9 for $L$ patterns $[t(22)=.40]$.

Procedure. Eight subjects participated in the experiment. All were undergraduates at Case Western Reserve who received course credit. Each experiment consisted of three 50-min sessions given on different (usually consecutive) days. Subjects were told that they would be shown two three-segment parts separated by a brief interval. Their task was to draw a sixsegment pattern, marking the three lines that occurred in the first exposure. The answer sheet contained 3 by 3 dot matrices, and subjects drew the patterns by connecting dots. They were required to always draw six lines and mark three lines, guessing when uncertain.

A Gerbrands three-channel tachistoscope was used to present the 24 patterns shown in Figure 1. Each trial consisted of a fixation field presented for $500 \mathrm{msec}$, a $500-\mathrm{msec}$ dark interval, a brief presentation of the first part, a dark interval of 40,80 , 120,160 , or $200 \mathrm{msec}$ duration, and a brief presentation of the second part followed by a dark interval. A part was presented for either $5 \mathrm{msec}$ (four subjects) or $10 \mathrm{msec}$ (four subjects) in order to determine whether slight differences in exposure duration would influence performance. The fixation field was 
Table 1

Ratings of Figural Goodness for the Whole Pattern, the Two Parts, and the Organization of the Parts within the Whole

\begin{tabular}{|c|c|c|c|c|c|c|c|c|}
\hline \multirow[b]{2}{*}{ Pattern } & \multicolumn{2}{|c|}{ Whole Pattern } & \multicolumn{2}{|c|}{ Common Part } & \multicolumn{2}{|c|}{ Different Part } & \multicolumn{2}{|c|}{ Organization } \\
\hline & $\mathrm{H}$ & $\mathrm{L}$ & $\mathrm{H}$ & L & $\mathbf{H}$ & $\mathrm{L}$ & $\mathrm{H}$ & $\mathbf{L}$ \\
\hline 1 & 2.8 & 5.0 & 4.7 & 4.7 & 3.6 & 4.6 & 2.3 & 2.4 \\
\hline 2 & 1.7 & 5.7 & 1.9 & 1.9 & 4.2 & 4.7 & 3.7 & 2.7 \\
\hline 3 & 2.2 & 5.8 & 1.8 & 1.8 & 1.9 & 3.1 & 6.9 & 4.7 \\
\hline 4 & 1.6 & 5.2 & 2.2 & 2.2 & 2.1 & 3.8 & 7.6 & 8.2 \\
\hline 5 & 1.8 & 4.3 & 2.0 & 2.0 & 2.1 & 3.5 & 9.2 & 9.1 \\
\hline 6 & 1.5 & 4.7 & 4.4 & 4.4 & 3.8 & 1.8 & 8.4 & 7.7 \\
\hline 7 & 2.1 & 5.1 & 2.6 & 2.6 & 4.1 & 5.2 & 3.6 & 4.2 \\
\hline 8 & 2.1 & 4.6 & 2.1 & 2.1 & 2.4 & 1.2 & 8.4 & 8.7 \\
\hline 9 & 2.7 & 4.8 & 1.6 & 1.6 & 5.0 & 4.2 & 2.7 & 5.4 \\
\hline 10 & 1.7 & 4.6 & 3.7 & 3.7 & 2.4 & 2.7 & 6.4 & 5.7 \\
\hline 11 & 1.4 & 5.8 & 3.5 & 3.5 & 3.8 & 6.1 & 6.6 & 3.0 \\
\hline 12 & 2.0 & 5.0 & 4.3 & 4.3 & 4.3 & 3.4 & 9.5 & 8.7 \\
\hline Average & 2.0 & 5.1 & 2.9 & 2.9 & 3.3 & 3.7 & 6.3 & 5.9 \\
\hline
\end{tabular}

Note-Figural goodness was judged on a 7-point scale ranging from 1 for well structured patterns to 7 for poorly structured patterns. The organization of parts was judged on an 11-point scale ranging from 0 for very poor organization to 10 for very good organization

a 3 by 3 dot matrix subtending a visual angle of $55 \mathrm{~min}$. The three-segment parts were presented within a dot matrix of the same size. The fixation field and the two parts were presented at a luminance of $4.4 \mathrm{ftL}$.

Each of the three sessions began with six practice trials on patterns that differed from the patterns used in the experiment. Each of the 24 patterns was presented twice at ISIs of 40,80 , 120,160 , and $200 \mathrm{msec}$. Two presentations were used in order that each part would occur once in the first exposure and once in the second exposure. There were a total of 16 presentations at each interval for each of the three sessions. The ISI did not change during a block of 16 trials. The order of presenting the blocks varied both across sessions and subjects.

\section{Results}

The effect of exposure duration was nonsignificant $(F<1)$, so the data from all eight subjects were combined. Figure 2 shows the percent of lines correctly

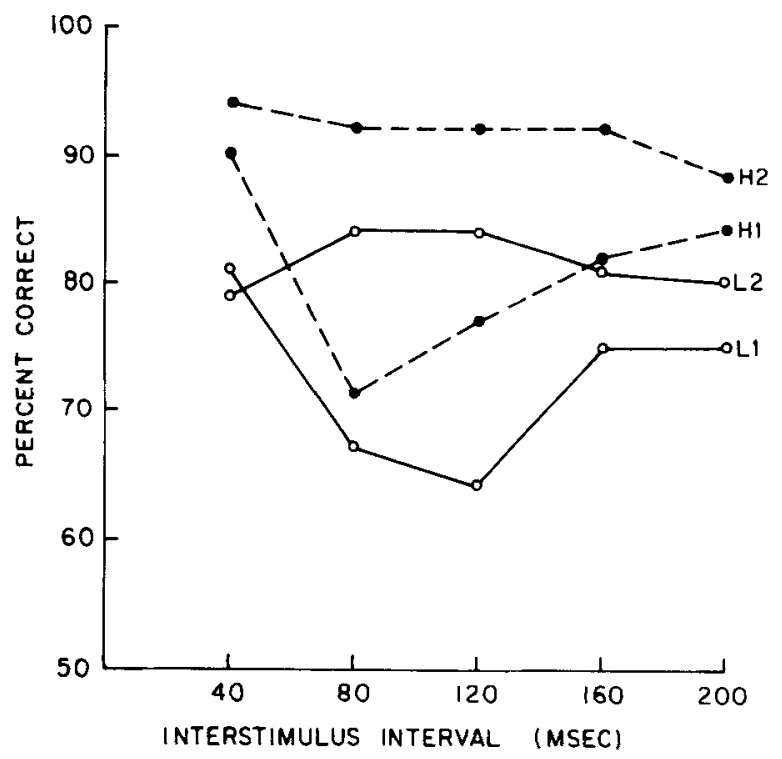

Figure 2. Percent of lines drawn correctly from the first part (1) and second part (2) for high-goodness (H) and low-goodness (L) patterns in Experiment 1. drawn at each of the five ISIs. The results are shown separately for the $\mathrm{H}$ and $\mathrm{L}$ patterns and for the firstexposure and second-exposure parts. An analysis of variance revealed that ISI had a significant main effect $[F(4,24)=7.73, p<.01]$ and interacted significantly with the order of presentation of the parts $[F(4,24)=9.55, p<.01]$. The order of presentation also had a significant main effect $[F(1,6)=13.04$, $p<.05]$, with the second part being drawn more accurately than the first part.

These findings are primarily the result of the substantial metacontrast effect illustrated in Figure 2. Accuracy in reproducing the lines from the first part was greatly affected by ISI, whereas accuracy on the second part was relatively independent of ISI. The metacontrast masking of the first part was greatest at an 80-msec ISI for $\mathrm{H}$ patterns and at a $120-\mathrm{msec}$ ISI for $\mathrm{L}$ patterns. One somewhat surprising finding was that the overall level of performance at a $40-\mathrm{msec}$ ISI was not substantially better than performance at a $200-\mathrm{msec}$ ISI. For H patterns, subjects correctly drew $91 \%$ of the lines at a $40-\mathrm{msec}$ ISI, compared to $87 \%$ at a $200-\mathrm{msec}$ ISI. For L patterns, subjects correctly drew $80 \%$ of the lines at a $40-\mathrm{msec}$ ISI and $77 \%$ at a $200-\mathrm{msec}$ ISI. Although these results were in the anticipated direction, a greater difference was expected.

The effect of figural goodness was significant $[F(1,6)=35.30, p<.01]$, and the better performance on $\mathrm{H}$ patterns was maintained over all ISIs. The scaling results from Experiment 1 revealed that although $\mathrm{H}$ and $\mathrm{L}$ patterns differed in the goodness ratings assigned to the whole patterns, they did not differ in the goodness or organization of the parts. One might, therefore, expect a Goodness by ISI interaction, with the difference between $\mathrm{H}$ and $\mathrm{L}$ patterns being more evident at short ISIs when the patterns are more likely to be perceived as wholes rather than as separate parts. However, there was little evidence for such an interaction $[F(4,24)=1.44, p>.05]$. Subjects were 
more accurate in drawing the $\mathrm{H}$ patterns at all ISI separations.

Another interaction of interest concerns whether the common part was reproduced more accurately when it occurred in a $\mathrm{H}$ pattern than in a $\mathrm{L}$ pattern. The overall results revealed that $86 \%$ of the lines in $\mathrm{H}$ patterns were correctly drawn, compared to $77 \%$ of the lines in $\mathrm{L}$ patterns. This difference was the result of an $8 \%$ difference on the common parts and a $10 \%$ difference on the different parts. Since this interaction was not significant $[F(1,6)=2.57]$, it seems reasonable to conclude that there was a significant context effect-the same part was more accurately reproduced when it occurred in a $\mathrm{H}$ pattern. ${ }^{1}$

In addition to drawing the six lines that composed the pattern, subjects were asked to always mark the three lines thai occurred in the first exposure. Figure 3 shows their ability to identify in which exposure the lines occurred. Only lines that were correctly drawn are included in these results. For example, if a subject marked two of three correct lines from the first exposure and one of two correct lines from the second exposure, his percentages would be $67 \%$ (upper curve) and $50 \%$ (lower curve). The results show that, at a 40 -msec ISI, $70 \%$ of the correct lines from the first exposure were marked as having occurred in the first exposure. This percentage increases to $82 \%$ at an 80 -msec ISI but does not improve with longer ISIs.

\section{Discussion}

The significant effect of figural goodness on performance is consistent with many previous research findings (Garner, 1974). It is somewhat surprising, however, that figural goodness did not interact with ISI. Figurally good patterns were reproduced more accurately even when it was more likely that the patterns were perceived as two three-segment parts rather than as a six-segment whole. Neither of the two most common explanations of figural goodness would seem capable of explaining the lack of interaction between figural goodness and ISI. One explanation proposes that figurally good patterns can be encoded more quickly then figurally poor patterns (see Bell \& Handel, 1976). An alternative explanation proposes that figurally good patterns are easier to remember (see Pomerantz, 1977). Both factors could have contributed to the advantage of figurally good patterns at a $40-\mathrm{msec}$ ISI, when it is likely that patterns could be perceived as a whole. However, since the average figural goodness of the parts did not differ for $\mathrm{H}$ patterns and $\mathrm{L}$ patterns, both explanations should predict no difference between $\mathrm{H}$ and $\mathrm{L}$ patterns when the patterns are encoded and reproduced from the two parts.

Another possible explanation of the results, the visual synthesis explanation (Palmer, 1977), would also have difficulty accounting for the advantage of $\mathrm{H}$ patterns over L patterns at a 200-msec ISI. According to this

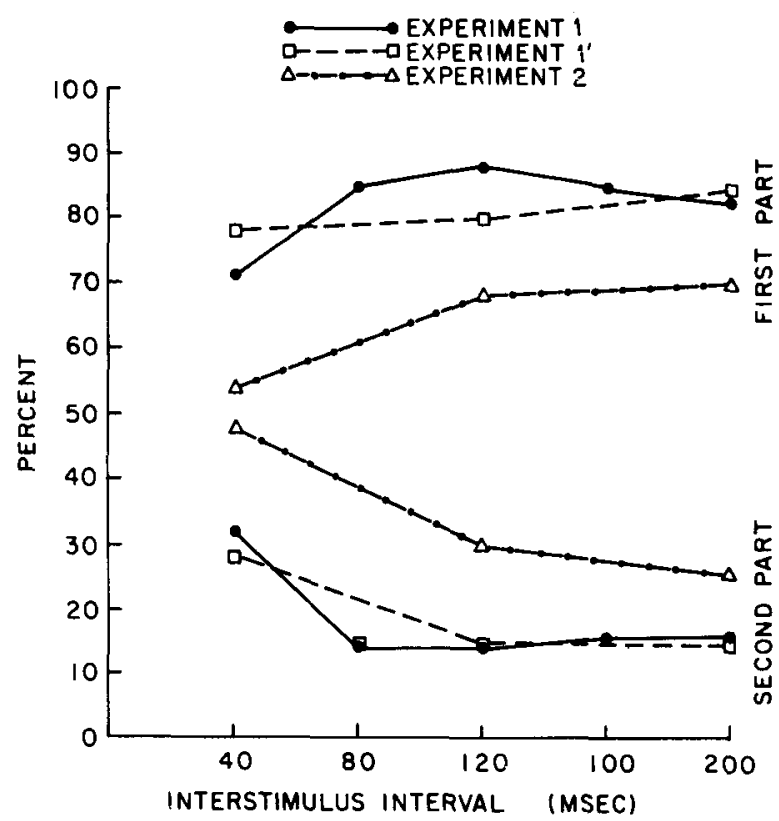

Figure 3. Percent of correctly drawn lines indicated as occurring in the first part.

explanation, subjects would synthesize the two parts to form the whole pattern, before attempting to reproduce the pattern. Palmer has shown that the difficulty of synthesizing a pattern is determined by whether it is divided into its most natural parts, but this variable was controlled across $\mathrm{H}$ and $\mathrm{L}$ patterns. The visual synthesis hypothesis could account for the present results if the figural goodness of the whole pattern determines the ease of synthesis in addition to the organization of the parts. Recent data by Thompson and Klatzky (1978) offer support for this interpretation. Irregular forms were very difficult to synthesize when compared with more familiar forms, such as triangles, trapezoids, and parallelograms. The $\mathrm{H}$ forms shown in Figure 1 are more familiar and may have been easier to synthesize.

There are other reasons, however, that the visual synthesis explanation is unattractive. Visual synthesis is a very difficult mental task, particularly when a pattern is not divided into its most natural parts (Palmer, 1977). Thompson and Klatzky (1978), in fact, questioned whether even the more familiar forms were completely synthesized, since subjects' ability to verify whether a part was present in a pattern was faster if the part matched one of the parts presented in the synthesis task. There are two aspects of the present paradigm that might further discourage subjects from attempting the kind of mental synthesis studied by Palmer and by Thompson and Klatzky. First, the synthesis would have to combine two parts that were recently presented but no longer present. In contrast, the task used by Palmer and by Thompson and Klatzky required the synthesis of parts that were present. Second, subjects in Experiment 2 were not required to 
synthesize the parts but were allowed to reproduce the pattern using whatever method they chose. If synthesis is a difficult mental task, and if its difficulty is increased by having to image the parts, there should be little incentive for subjects to synthesize the whole pattern before reproducing it.

The interpretation we favor proposes a response bias for familiar or figurally good forms. The response-bias hypothesis states that subjects attempt to construct a familiar or figurally good pattern when they are uncertain about which lines were presented. This interpretation is consistent with Bear's (1973) research on predicting the elements of a pattern. He hypothesized that if good figures are characterized by well organized elements, each element of a good pattern should be strongly suggested or implied by the other elements. This close relationship should not exist for the elements of a poor figure. This differential predictability was tested by showing subjects a four-dot subpattern and asking them to fill in the missing fifth dot. The dots comprising a figurally good five-dot pattern were generally more predictable than the dots comprising a figurally poor pattern. It should be noted that a bias toward drawing figurally good patterns should exist at all ISIs and could therefore account for the data in Experiment 1.

\section{EXPERIMENT 2}

The purpose of Experiment 2 was to investigate the organization of the parts within the pattern as an independent variable. The constraint that $H$ and $L$ patterns share a common part in Experiment 1 precluded the systematic construction of different part structures for each pattern. Subjects in a scaling task were asked to rate the organization of alternative parts based on the technique used by Palmer (1977). Pairs of patterns differing in levels of organization were then used in a reproduction task.

\footnotetext{
Method

Stimuli. Two scaling sessions were needed to select the stimuli for Experiment 2. The purpose of Experiment 2 was to examine the effect of dividing a pattern into different combinations of parts, having either a high or a low level of organization. Each of the original 54 patterns was divided into three different two-part combinations, with the two parts appearing to the right of the whole pattern. The three partitions of a pattern were constructed to represent a high, medium, or low level of organization following the procedure used by Palmer (1977). The high level of organization was based on the most frequently constructed partition of the pattern found in Experiment 1. Subjects were asked to judge how obvious or natural the parts were within the pattern, using an 11-point scale ranging from 0 for a very bad organization to 10 for a very good organization. Thirteen undergraduates made a total of 162 judgments. The second session asked a different group of 26 undergraduates to rate the figural goodness of the threesegment parts created in the previous session. Subjects rated the 224 parts on a 7 -point scale. The parts were presented in a
}

random order and were not shown within the context of the whole pattern.

The ratings were used to select 18 pairs of patterns for use in Experiment 2. Each pair consisted of the same pattern broken down into either a high (natural parts) or a low (unnatural parts) level of organization. Nine pairs were figurally good patterns and nine pairs were figurally poor patterns, based on the ratings from Experiment 1 . The criterion for selecting patterns was that the ratings on both figural goodness of the whole pattern and the organization of the parts be at the extreme ends of the scale.

The stimuli consisted of 18 pairs of patterns. Nine pairs were figurally good patterns (average rating $=1.90$ ) and nine pairs were figurally poor patterns (average rating $=5.28$ ). Each pair consisted of the pattern divided into a high and a low level of organization. The two parts representing the high level of organization were the most frequent divisions made by the subjects in Experiment 1. The average organization rating was 9.13 for figurally good patterns and 8.72 for figurally poor patterns. The two parts representing the low level of organization had at least one line that was not connected to the other two lines in a part. The number of unconnected lines ranged from 1 to 6 , with an average of 3.2 for figurally good patterns and 1.8 for figurally poor patterns. In contrast, all lines were connected in the parts comprising the high level of organization. The average rating for the low-organization patterns was 2.71 for figurally good patterns and 3.47 for figurally poor patterns. Figure 4 shows an example of the four types of patterns. ${ }^{2}$

Procedure. The procedure was similar to that used in Experiment 1. Each trial consisted of a 500-msec presentation of the fixation field (the dot matrix), a 500-msec dark interval, a 7 -msec presentation of the first part, a variable dark interval, a 7-msec presentation of the second part, and a dark interval. The ISI was 40,120 , or $200 \mathrm{msec}$. Subjects were asked to always draw six line segments and mark the three that occurred first.

Seven subjects participated in the three sessions of the experiment. The 36 stimuli were presented twice at each ISI with each of the two parts occurring once during the first exposure and once during the second exposure. A session consisted of one block of 24 trials for each of the three ISIs. The order of the three blocks varied both across subjects and sessions. Stimuli occurred in a random order under the constraint that the same stimulus could not appear on successive trials.

\section{Results}

An analysis of variance revealed that figural good-
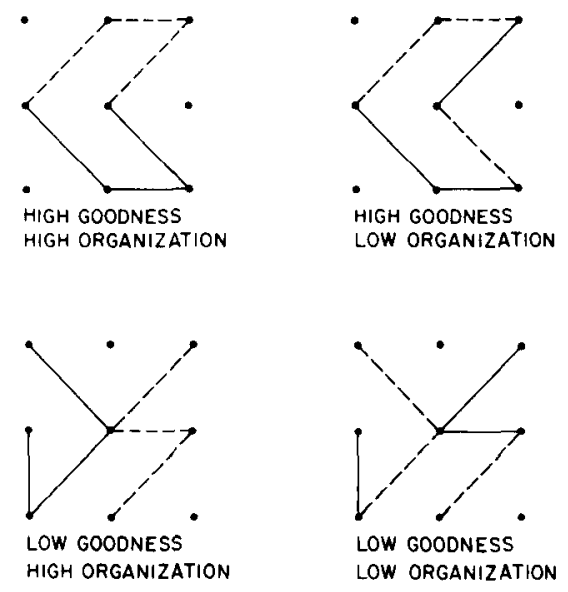

Figure 4. Examples of patterns used in Experiment 2. 
ness $[F(1,6)=22.90, p<.01]$, organization of parts $[F(1,6)=47.18, \quad p<.01], \quad$ order of presentation $[F(1,6)=11.50, p<.05]$, and $\operatorname{ISI}[F(2,12)=10.10$, $\mathrm{p}<.01]$ had significant effects on performance. Figural goodness did not interact with ISI $(F<1)$, supporting the results from Experiment 1 in which the better performance on figurally good patterns was maintained over all intervals of part separation.

The results of the experiment are shown in Figure 5. Figure 5a shows subjects' performance on patterns that were judged to have a high level of organization. Although the level of performance is slightly lower, the effects of the experimental variables are very similar to the effects obtained in Experiment 1 (see Figure 2). There is a slight improvement in accuracy when the parts are separated by $40 \mathrm{msec}$ rather than $200 \mathrm{msec}$. Subjects correctly reproduced $76 \%$ of the lines from figurally good patterns at a $40-\mathrm{msec}$ separation, compared to $71 \%$ at a $200-m s e c$ separation. They were correct on $63 \%$ of the lines from figurally poor patterns at a 40 -msec separation, compared to $60 \%$ at a $200-\mathrm{msec}$ separation.

The relative lack of effect of ISI on organizing the natural parts of a pattern changes dramatically when the pattern is poorly organized. Figure $5 \mathrm{~b}$ shows that an ISI of $120 \mathrm{msec}$ or greater produces a large decrement in performance when subjects attempt to reproduce the first part of a poorly organized pattern. A significant Organization by Interval interaction $[F(2,12)=10.68$, $\mathrm{p}<.01]$ indicates that varying the ISI mainly influences poorly organized patterns. The decline in performance with increasing ISI is most apparent for the first part, as revealed by the interaction between organization and order of presentation $[F(1,6)=6.21, p<.05]$. There was no interaction between figural goodness and the organization of the parts $(F<1)$. A poorly organized pattern has similar effects on figurally good and figurally poor patterns, although performance is better on the former (see Figure $5 b$ ).

One interesting finding in Experiment 2 requires comparing Figures $5 \mathrm{a}$ and $5 \mathrm{~b}$ at the $40-\mathrm{msec}$ ISI. Although the organization of the pattern had a highly significant main effect on performance, it had little effect at a 40-msec ISI. For figurally good patterns, subjects correctly drew $76 \%$ of the lines from well organized patterns and $74 \%$ of the lines from poorly organized patterns. For figurally poor patterns, the subjects correctly drew $63 \%$ of the lines from well organized patterns and $61 \%$ of the lines from poorly organized patterns. These results could be explained by the assumption that subjects were perceiving the pattern as a whole at this ISI, so the part structure of the pattern was unimportant.

Figure 3 shows subjects' ability to identify which line segments occurred in the first exposure. Temporal discrimination was poorer in Experiment 2 than it was in Experiment 1. Performance was close to the 50\% chance level at a 40-msec ISI but improved with longer delays between the two parts. It is not entirely clear why temporal discrimination and reproduction accuracy were at lower levels in Experiment 2. One possibility is that there were more pattems in Experiment 2, and the patterns were seen less often, making it more difficult to learn the pattern set. Some of the patterns were different from those used in Experiment 1 and may have been more difficult. Another possibility is simply that there were individual differences among the two groups of subjects.
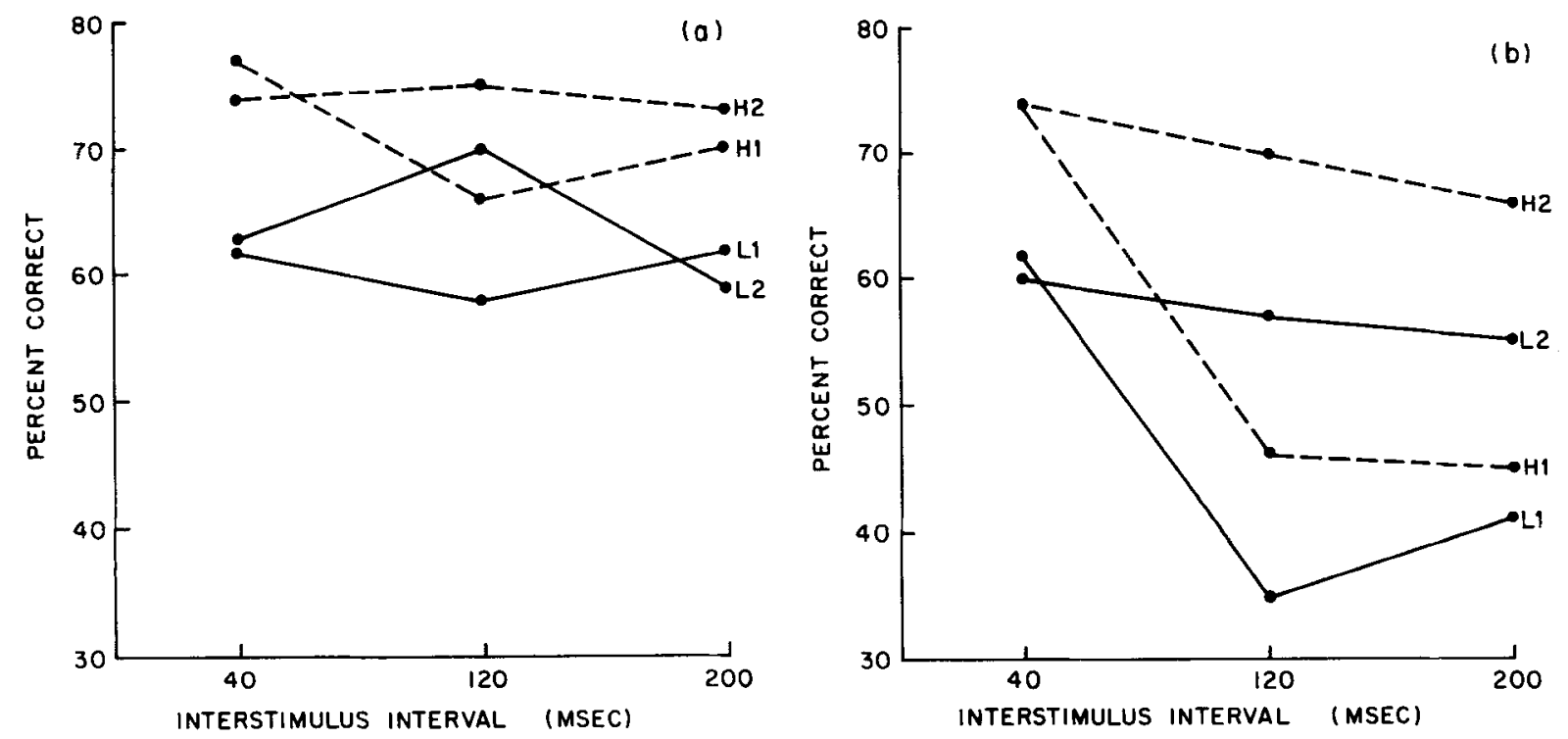

Figure 5. Percent of lines drawn correctly from the first part (1) and second part (2) for high-goodness (H) and low-goodness (L) patterns in Experiment 2. (a) High organization. (b) Low organization. 


\section{Discussion}

The lack of interaction between figural goodness and ISI replicates the results of Experiment 1. The slight advantage of the $40-\mathrm{msec}$ ISI over the $200-\mathrm{msec}$ ISI was confirmed for patterns having a high level of organization (Figure 5a). For figurally good patterns, reproduction accuracy was $5 \%$ higher at a $40-\mathrm{msec}$ ISI than at a $200-\mathrm{msec}$ ISI. For figurally poor patterns, there was a $3 \%$ advantage at the $40-\mathrm{msec}$ ISI. The results are almost identical to the $4 \%$ advantage for figurally good patterns and the 3\% advantage for figurally poor patterns found in Experiment 1 . Thus in all four cases, the probability of reproducing the lines of a pattern at a $40-\mathrm{msec}$ ISI ranged from $3 \%$ to $5 \%$ higher than at a $200-\mathrm{msec}$ ISI.

The most salient finding in Experiment 2 was that the temporal separation of the parts dramatically affects performance when a pattern is poorly organized. Figure 5b shows that accuracy in reproducing the first part declines sharply with increasing ISI. It is likely that subjects had great difficulty in remembering the first part after the second part was presented. The parts in the low-organization condition contained many unconnected lines, which should be more difficult to remember. In contrast, all lines were connected in the parts of high-organization patterns, and most of the lines were connected in the parts of the patterns used in Experiment 1 (see Figure 1). Thus, the number of unconnected lines would seem to be an important variable in determining subjects' ability to reproduce a pattern as a function of the temporal separation of its parts.

\section{GENERAL DISCUSSION}

Our intention in this concluding section is to summarize the principal findings, offer a tentative interpretation, and suggest experimental paradigms for testing our interpretation.

\section{Summary of the Results}

Figural goodness. Figurally good patterns were reproduced more accurately than figurally poor patterns at all ISIs. Better performance on figurally good patterns occurred even at intervals (such as $200 \mathrm{msec}$ ) when subjects perceived two separate parts rather than the whole pattern. This occurred even when the average organization of the parts did not differ between the two sets of patterns (Experiments 1, 1', and 2).

Context. A three-segment part was reproduced more accurately when it occurred in a $\mathrm{H}$ pattern, than when it occurred in a $\mathrm{L}$ pattern (Experiments 1 and $\left.1^{\prime}\right)$. However, performance was best when a part was presented alone (Experiment 1').

Organization of the parts. The organization of the parts did not influence performance at a 40-msec ISI. However, reproduction accuracy of the first part declined dramatically with increasing ISI for poorly organized parts. Highly organized parts were affected only slightly, but consistently, with increasing ISI.

Interstimulus interval. The most interesting comparison involved the extreme intervals since metacontrast masking occurred at the intermediate intervals. For the patterns used in Experiment 1 and the patterns having well organized parts in Experiment 2, there was a $3 \% 5 \%$ improvement in performance at a $40-\mathrm{msec}$ ISI over performance at a $200-\mathrm{msec}$ ISI. For patterns having poorly organized parts, reproduction of the first part was either $21 \%$ (for figurally poor patterns) or $29 \%$ (for figurally good patterns) higher at the $40-\mathrm{msec}$ ISI.

\section{A Tentative Explanation}

The present series of experiments was designed to test how several task parameters would influence performance on a sequential part task. Further research is necessary to determine the psychological processes used by subjects to perform the task. We would like, however, to offer what we feel is a plausible explanation and suggest some extensions of the paradigm to test the explanation.

The ISIs were chosen in an attempt to create a task in which perception would continuously change from perception of a whole pattern at a $40-\mathrm{msec}$ ISI to the perception of two separate parts at a $200-\mathrm{msec}$ ISI. Although the change may occur along a continuum, metacontrast masking at $80-120 \mathrm{msec}$ makes the intermediate intervals less interesting, so we have mainly focused our attention on the two extreme points, $40 \mathrm{msec}$ and $200 \mathrm{msec}$.

Several aspects of the data are consistent with the hypothesis that subjects perceive the pattern as a whole at a 40-msec separation and as two separate parts at a 200 -msec separation. The clearest evidence is provided by comparing the low- and high-organization conditions of Experiment 2. At a $40-\mathrm{msec}$ ISI, it did not make any difference whether the pattern was divided into well organized or poorly organized parts. This finding is consistent with the proposal that the pattern was perceived as a whole. However, at a $200-\mathrm{msec}$ ISI, patterns that were divided into well organized parts were reproduced less well than patterns that were divided into poorly organized parts. These data produced a highly significant interaction between organization and ISI.

The results of Eriksen and Collins (1967) also support the interpretation that a pattern becomes increasingly difficult to perceive as a whole as the ISI increases from 0 to $100 \mathrm{msec}$. Their stimuli consisted of pairs that were perceived as a random collection of dots when viewed alone or as a three-letter nonsense syllable when superimposed. A uniform decline in subjects' ability to report the nonsense syllable with increasing temporal separation suggested an increasing tendency to perceive the two exposures as separate rather than superimposed. 
If we assume that the above explanation is correct, we can then ask if there is any advantage in perceiving the entire pattern rather than its separate parts. Experiments 1 and 2 suggest that when patterns are at least moderately well organized, perceiving the whole pattern produces a slight but consistent advantage, causing a 3\%-5\% improvement in performance. It is likely that perception of the entire pattern allows for more efficient grouping and chunking of line segments, making it easier to remember the pattern. The results of Experiment 2 suggest that perceiving the separate parts produces a dramatic decline in performance when the parts are poorly organized (Figure 5b). One distinguishing characteristic of this particular set of patterns is the number of unconnected lines. There was an average of 2.5 unconnected lines in the two parts, compared to 0 unconnected lines for the highorganization patterns. There was a substantial range in the average organization ratings assigned to the patterns used in Experiment 1, but the number of unconnected lines averaged only 3 for the two parts.

The poor performance in reproducing the poorly organized parts was caused by subjects' difficulty in reconstructing the first part. This finding is consistent with the results of Hines and Smith (1977), who demonstrated that an attended random shape caused substantial retroactive interference in identifying a random shape that occurred $150-300 \mathrm{msec}$ earlier. If subjects did not have to identify the second shape, there was virtually no interference in identifying the first shape. Our results indicate that a second pattern makes it more difficult to reproduce the first pattern (Experiment $1^{\prime}$ ), but the magnitude of the effect is greatly influenced by the structure of the two parts (Experiment 2).

Perhaps our most surprising finding was the lack of interaction between the figural goodness of the whole pattern and ISI. Subjects were more accurate in reproducing figurally good patterns even when perceived as parts. The result was surprising because the structure and organization of the parts did not differ between figurally good and figurally poor patterns. We considered several possible explanations of this finding when discussing the results of Experiment 1 and selected the one postulating a response bias in favor of figurally good patterns. The response-bias hypothesis states that subjects fill in missing features by attempting to construct a figurally good pattern.

To summarize our interpretation of the results, the effect of increasing the ISI from $40 \mathrm{msec}$ to $200 \mathrm{msec}$ is to cause subjects to perceive the pattern as a six-segment whole at one extreme (a 40-sec separation) and as two three-segment parts at the other extreme $(200 \mathrm{msec})$. Following the application of Rumelhart's (1970) feature recognition model to metacontrast masking, we believe that feature recognition occurs simultaneously from the two parts at short separations ( $40 \mathrm{msec}$ or less), but the features from the first part are recognized before the second part is presented at longer separations ( $200 \mathrm{msec}$ or more). At intermediate separations $(80-120 \mathrm{msec})$, subjects are still in the process of forming a structural description of the first part when the second part occurs. Recognizing the features of the second part therefore interferes with forming a description of the first part, causing metacontrast masking. Because the line segments in a six-segment whole pattern can be more efficiently grouped and chunked than the line segments in the two parts, reproduction accuracy is higher at a $40-\mathrm{msec}$ ISI than at a $200-\mathrm{msec}$ ISI. If the organization of the pattern is divided into poorly organized parts, retroactive interference caused by the second part makes it extremely difficult to remember the first part. When subjects perceive two separate parts, they do not mentally synthesize the parts before attempting to reproduce the whole. They therefore construct a six-segment pattern from the two parts but are biased toward constructing a figurally good pattern when uncertain about some of the features.

\section{Further Research}

There are a number of possible extensions of the current paradigm that could be used to test our tentative explanation. The visual synthesis paradigm offers a methodology for examining whether a person has encoded the pattern as a whole or as two separate parts. Following the technique designed by Palmer (1977), subjects could be shown a six-segment test pattern after seeing the two parts. The test pattern would be either similar or identical to the pattern formed from the parts and the task would be to decide if the two patterns were identical. A control condition would present a six-segment whole pattern followed by the test pattern. We would expect that accuracy and reaction times for the whole and part conditions would be similar at a 40-msec ISI, but subjects' performance would decline at a 200-msec ISI because subjects would not know what the whole pattern looked like.

Another promising paradigm would use two line segments as the test pattern. This paradigm was designed by Cunningham (Note 1) to build a tree structure showing the relations among the line segments of a pattern. Cunningham used the time needed to verify that two line segments were contained within a pattern as a measure of the "distance" between the two line segments. For example, line segment a would be equidistant from lines $b$ and $c$ if reaction times to pairs $a b$ and ac were the same. Now divide the pattern into two parts such that lines $a$ and $b$ belong to the same part, but $\mathrm{c}$ belongs to a different part. If the two parts are perceived as a whole pattern, reaction times to pairs ab and ac should remain the same. However, if the pattern were encoded as two separate parts, Thompson and Klatzky's (1978) results would imply that the verification time for $a b$ should be less than for ac. 
Systematic testing and the use of Cunningham's graph techniques should enable one of examine how the memory representation changes as a function of the ISI.

One possible test of the response-bias interpretation, proposed to explain the lack of an interaction between figural goodness and ISI, would be to delete a line segment on some of the trials. Thus, subjects would expect and draw a six-segment pattern but would be shown only five lines on some trials. We would expect on the basis of Bear's (1973) findings that subjects would correctly draw the missing segment more often when a figurally good pattern was presented than when a figurally poor pattern was presented.

In conclusion, we have attempted to explore the temporal organization of line patterns by examining the relations among figural goodness, organization of the parts, and the ISI. We proposed a tentative explanation of the findings and suggested some extensions of the paradigm to test our explanation.

\section{REFERENCE NOTE}

1. Cunningham, J. P. Memory for simple visual patterns. Paper presented at the annual meeting of the Psychonomic Society, Washington, D.C., November 1977.

\section{REFERENCES}

BEAR, G. Figural goodness and predictability of figural elements. Perception \& Psychophysics, 1973, 13, 32-40.

Bell, H. H., \& Handel, S. The role of pattern goodness in the reproduction of backward masked patterns. Journal of Experimental Psychology: Human Perception and Performance, 1976, 2, 139-150.

Eriksen, C. W., \& Collins, J. F. Some temporal characteristics of visual pattern perception. Journal of Experimental Psychology, 1967, 74, 476-484.

GARNER, W. R. The processing of information and structure. Potomac: Erlbaum, 1974.

Garner, W. R., \& Clement, D. E. Goodness of pattern and pattern uncertainty. Journal of Verbal Learning and Verbal Behavior, 1963, 2, 446-452.

HABER, R. N. Visual perception. In Annual review of psychology. Palo Alto, Calif: Annual Reviews, Inc., 1978.

Hines, D., \& SMith, S. Recognition of random shapes followed at varying delays by attended or unattended shapes, digits, and line grids. Journal of Experimental Psychology: Human Learning and Memory, 1977, 3, 29-36.

Hochbe RG, J. E. Perception (2nd ed.). Englewood Cliffs, N.J: Prentice-Hall, 1978.

McFarland, J. H. Sequential part presentation: A method for studying visual form perception. British Journal of Psychology, $1965,56,439-446$.
Palmer, S. E. Hierarchical structure in perceptual representation. Cognitive Psychology, 1977, 9, 441-474.

Pomerantz, J. R. Pattern goodness and speed of encoding. Memory \& Cognition, 1977, 5, 235-241.

Pomerantz, J. R., Sager, L. C., \& Stoever, R. J. Perception of wholes and of their component parts: Some configural superiority effects. Journal of Experimental Psychology: Human Perception and Performance, 1977, 3, 422-435.

REED, S. K. Schemes and theories of pattern recognition. In E. C. Carterette \& M. P. Friedman (Eds.), Handbook of perception (Vol. 9). New York: Academic Press, 1978.

Rumelhart, D. E. A multi-component theory of perception of briefly exposed stimulus displays. Journal of Mathematical Psychology, 1970, 7, 191-218.

Thompson, A. L., \& Klatzky, R. L. Studies of visual synthesis: Integration of fragments into forms. Journal of Experimental Psychology: Human Perception and Performance, 1978, 4 244-263.

Williams, A., \& Weisstein, N. Line segments are perceived better in a coherent context than alone: An object-line effect in visual perception. Memory \& Cognition, 1978, 6, 85-90.

\section{NOTES}

1. Recent research has indicated that, under certain conditions, a part is recognized better when it appears in the context of a pattem than when it appears by itself (Pomerantz, Sager, \& Stoever, 1977; Williams \& Weisstein, 1978). This finding was tested in a slightly modified version of Experiment 1 using a different group of eight subjects. The procedure was identical except that only three ISIs were used $(40,120$, and $200 \mathrm{msec}$ ) and subjects had to reproduce only a single part on some trials (the fixation field replaced by the second part). The context produced a significant main effect $[F(2,14)=18.64$, $\mathrm{p}<.011$. When the part occurred alone, $95 \%$ of the lines were drawn correctly, compared to $85 \%$ when it occurred as part of a high-goodness whole and $78 \%$ when it occurred as part of a low-goodness whole. A Newman-Keuls analysis revealed that presenting a part alone differed significantly at the .01 level from presenting it in either context, and presenting it in a goodfigure context differed at the .05 level from presenting it in a poor-figure context. The latter finding confirms the facilitating effect of a good-pattern context found in Experiment 1. The effect of context did not interact with $\operatorname{ISI}[F(4,28)=1.62]$, so the superior performance in reproducing a part that occurred alone was not caused by metacontrast masking when both parts occurred. Subjects' ability to temporally discriminate the two parts, when both occurred, is shown in Figure 3 as Experiment 1'.

2 . The average figural goodness rating of parts was 2.88 for high-organization patterns and 4.77 for low-organization patterns. In general, well organized patterns tend to consist of figurally good parts and poorly organized patterns tend to consist of figurally bad parts. The current research does not at tempt to independently evaluate these two variables.

(Revision accepted for publication March 13, 1979.) 